\title{
Killing me softly: microparticles target deformable cells
}

\author{
Cell Research (2016) 26:637-638. doi:10.1038/cr.2016.54; published online 10 May 2016
}

Mechanical deformability is known to increase during cancer progression. Now Ma et al. identify a therapeutic strategy that targets deformable cells by microparticle-based drug delivery.

Cancer cells tend to be softer than normal cells $[1,2]$. Cells with increasing malignant potential [3-4], and expressing activated oncogenes such as Kras [1], have reduced cortical tension and increased mechanical deformability relative to control cells. The relative softness of cancer cells may promote squeezing through small spaces during metastasis [3], or could promote cell survival [5]. Tumor-initiating cells, sometimes referred to as cancer stem cells or tumor-repopulating cells (TRCs), which are most able to initiate cancer growth, may be even more deformable than other cancer cells $[6,7]$. However, while it is clear that mechanical changes accompany carcinogenesis, strategies to take advantage of this difference between cancer and normal cells, a potential Achilles' heel, have remained few. But now, some really good ideas are starting to emerge.

It may be possible to simply increase tension in cancer cells and reverse the effects of mechanical deformability. Activation of myosin light chain kinase, Rho-kinase, or RhoA within tumorinitiating cells in glioma reduced cell invasion and also extended survival in mice [8], suggesting that increasing cell tension could be of therapeutic benefit. Notably, a recent chemical screen for modulators of cell tension identified a proof-of-principle compound called 4-HAP that increases tension by targeting myosin II [9]. Treatment of pancreatic cancer cells with 4-HAP increased cortical tension to levels similar to non-tumor cells, and inhibited cancer cell migration and invasion, suggesting a potential therapeutic strategy. Alternatively, it may be possible to identify therapeutic approaches that take advantage of the differences in tension between cancer and normal cells. In a recent paper in Cell Research, Ma et al. [10] report a potential therapeutic strategy that does just that: it exploits the lowered tension of cancer cells and TRCs.

Using apoptotic tumor cell-derived microparticles (MPs), previously published by the Huang lab as efficient cytotoxic drug-delivery vehicles [11], the authors treated selected TRCs from cancer cell lines and parental cell populations and found that while drugloaded MPs were generally cytotoxic, they killed TRCs even more efficiently than unsorted cancer cells. TRCs are often thought to be drug-resistant [12]. Indeed, when cytotoxic drugs were administered in the absence of MPs, they killed TRCs less efficiently than parental cells. Thus, it seems that the MP loading of commonly used chemotherapeutic drugs (cisplatin, doxorubicin, and methotrexate) can overcome drug resistance to eliminate TRCs.

Why do drug-loaded MPs work so well? There may be at least two reasons. First, the authors find that mechanical deformability, a property of TRCs, promotes efficient MP uptake. Increasing tension by treatment with the actin polymerizing compound jasplakinolide, or inhibiting tension by treatment with the myosin II inhibitor blebbistatin, reduced or enhanced MP uptake, respectively. Thus, TRCs may be particularly susceptible to this mode of drug delivery due to their mechanical properties. Second, once ingested, MPs blocked drug export, an activity that is well-known to lead to drug resistance. The combined effects of enhanced uptake of drug-loaded MPs into TRCs and increased drug retention promote the MP-mediated killing of otherwise drug-resistant cells.

The authors demonstrate the potential therapeutic efficacy of their approach by using a mouse model of lung carcinoma, where methotrexateloaded MPs delivered intravenously reduced Lewis carcinoma lung tumor burden better than treatment with free drug. Importantly, the administration of cisplatin-loaded MPs by intrathoracic injection to end-stage human lung cancer patients induced dramatic clearance of cisplatin-resistant cells from malignant fluids, with $>95 \%$ of cancer cells cleared after a 7-day treatment, suggesting that this approach has therapeutic potential.

The findings by Ma and colleagues [10] open the door to a new potential therapeutic approach that takes advantage of the mechanical changes that accompany carcinogenesis. Whether solid tumors can be targeted in this manner by intravenous injection of MPs in human patients, and which types of cancers are susceptible, are important questions for further study. Interestingly, MPs appear to have unique effects on targeted cells that may not be shared by synthetic micro- or nanoparticles [11]. For example, the administration of MPs can affect drug retention and nuclear entry in trans, where drug-free MPs promote the nuclear uptake of soluble drugs, 


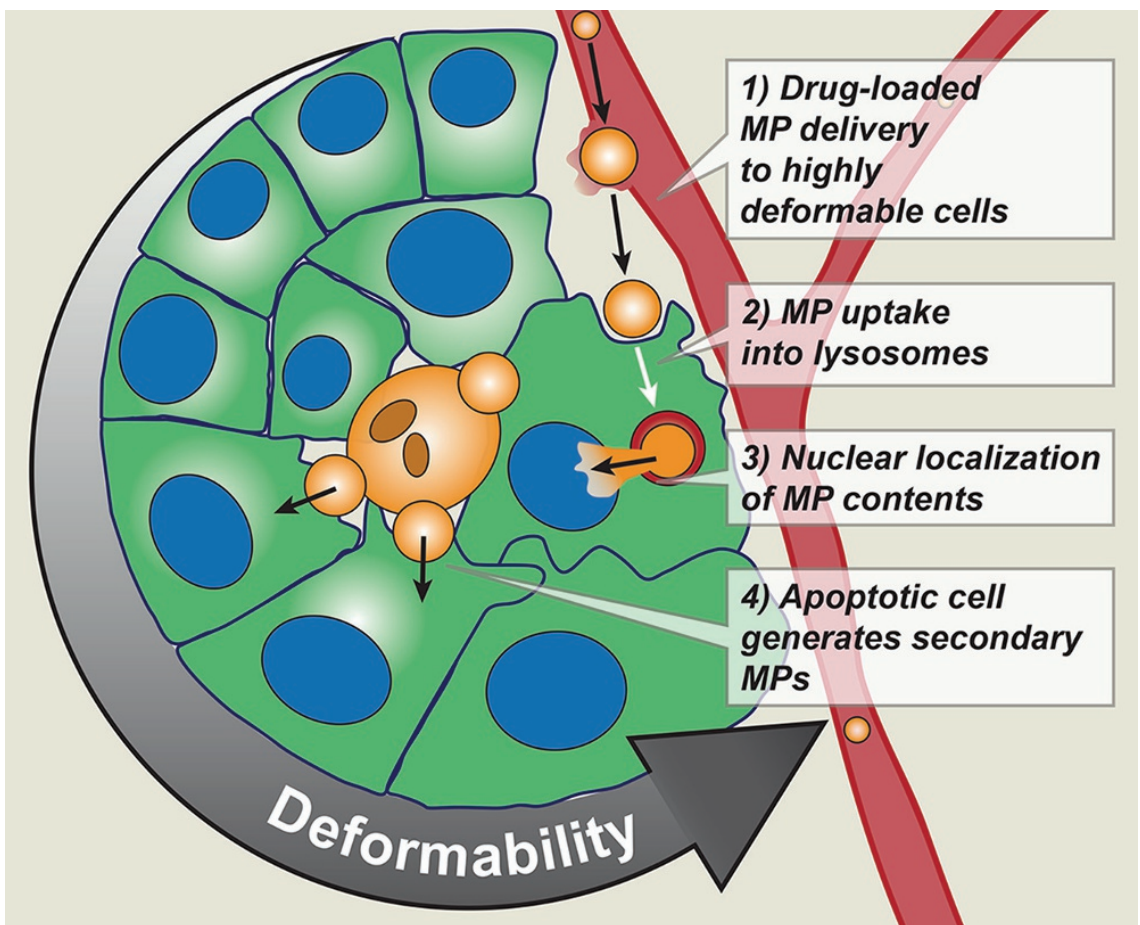

Figure 1 Chemotherapy drug-loaded MPs target soft TRCs. 1) Tumor cell-derived MPs (orange spheres) are delivered to tumor sites through leaky vasculature. MPs are preferentially taken up by TRCs due to the increased deformability of these cells. 2) MPs and associated drugs traffic to lysosomes (red sphere). 3) Contents of MPs enter the nuclei of targeted cells, possibly through dynein-mediated retrograde trafficking. 4) Following apoptotic cell death induced by MPs, apoptotic bodies act as secondary MPs to carry chemotherapeutic drugs to neighboring cells.

potentially due to effects on drug transporter expression or nuclear entry [10]. Whether the cellular origin, lipid content, or otherwise specific composition of MPs is important for their cytotoxic effects will be interesting to examine in future studies. Identifying the mechanisms controlling how MPs are taken into cells, potentially trafficked, and how they deliver their toxic cargo into the nucleus, will also be important in cells that undergo apoptosis as a result of this strategy [11]. In this manner, the death of cancer cells that ingest MPs, perhaps proximal to tumor vasculature that is sufficiently leaky, could spread to others not initially reached by intravenous delivery. Like a domino, the first cell to fall could trigger a therapeutic chain reaction that eliminates cancer (Figure 1).

Jens Hamann
Michael Overholtzer

${ }^{I}$ Cell Biology Program and Louis V Gerstner $J r$ Graduate School of Biomedical Sciences, Memorial Sloan Kettering Cancer Center, New York, NY 10065, USA

Correspondence: Michael Overholtzer

E-mail: overhom1@mskcc.org

\section{References}

1 Anderson KW, Li WI, Cezeaux J, et al. Cell Biophys 1991; 18:81-97.

2 Cross SE, Jin YS, Rao J, et al. Nat Nanotechnol 2007; 2:780-783.

$3 \mathrm{Xu} \mathrm{W,} \mathrm{Mezencev} \mathrm{R,} \mathrm{Kim} \mathrm{B,} \mathrm{et} \mathrm{al.} \mathrm{PLoS} \mathrm{One}$ 2012; 7:e46609.

4 Ward KA, Li WI, Zimmer S, et al. Biorheology 1991; 28:301-313.

5 Sun Q, Luo T, Ren Y, et al. Cell Res 2014; 24:1299-1310.

6 Sun J, Luo Q, Liu L, et al. J Biomech 2016; 49:45-52.

7 Zhang W, Kai K, Choi DS, et al. Proc Natl Acad Sci USA 2012; 109:18707-18712.

8 Wong SY, Ulrich TA, Deleyrolle LP, et al. Cancer Res 2015; 75:1113-1122.

9 Surcel A, Ng WP, West-Foyle H, et al. Proc Natl Acad Sci USA 2015; 112:1428-1433.

10 Ma J, Zhang Y, Tang K, et al. Cell Res 2016 May 10. doi: 10.1038/cr.2016.53

11 Tang K, Zhang Y, Zhang H, et al. Nat Commun 2012; 3:1282.

12 Vinogradov S, Wei X. Nanomedicine 2012; 7:597-615. 